\title{
Numerical Analysis on Reusable Rocket Aerodynamics with Reduced-Yaw-Force Configurations
}

\author{
By Ayano InAtomi, ${ }^{1)}$ Keiichi KitAmura, ${ }^{1)}$ and Satoshi NonAKA ${ }^{2)}$ \\ 1) Yokohama National University, Yokohama, Japan \\ 2) Institute of Space and Astronautical Science, JAXA, Sagamihara, Japan
}

(Received June 30th, 2017)

\begin{abstract}
It is known that aerodynamic characteristics of a slender body vary substantially at high angles-of-attack (AoAs), and then, will have strong impacts on its flight. For, example, the yaw force makes flight unstable. In this study, we investigated the relation between the yaw force and the configuration, and details of flowfield around the slender-bodied-vehicle numerically. The configuration consisting of "nose cone" and "square aftbody" parts was employed as the baseline, and then, compared with other three configurations having different fineness ratios. According to our computed results, in the case of 50 degrees of AoA, the longer the model became, the more asymmetry appeared: yaw force and asymmetry were found to be attributed not only to the length of the body, but also to the nose bluntness. On the contrary, in the case of 140 degrees, the shorter the model became, the more asymmetry appeared. Furthermore, the large nose bluntness increased $\mathrm{CY}_{\mathrm{Y}}$. Interestingly, this trend is totally opposite to that observed at 50 degrees. It had been considered that the large nose bluntness and the small fineness ratio can reduce asymmetry and $\mathrm{C}_{Y}$, however, this study showed that it is not true in the case over 90 degrees, due to complex wake flow structure discovered in the present numerical simulations.
\end{abstract}

Key Words: Aerodynamic Characteristics, CFD, Reusable Rocket, Yaw Force, Asymmetric Vortices

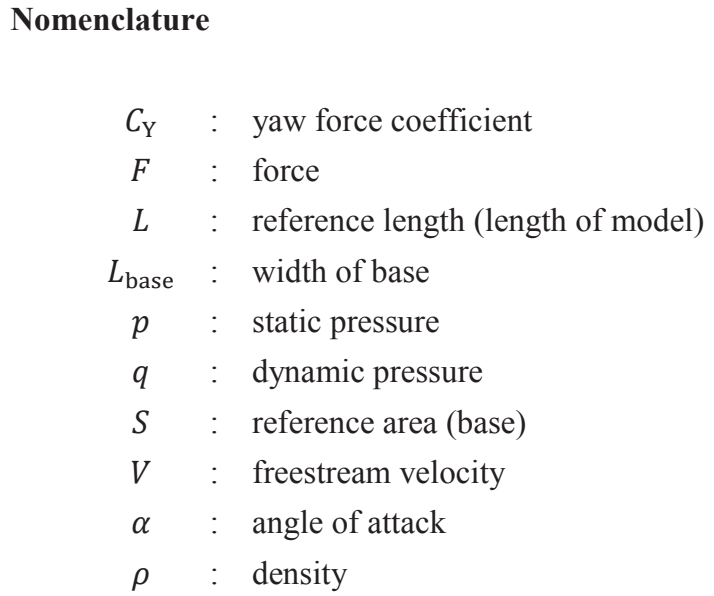

Subscripts

$$
x, y, z \quad: \quad \mathrm{x}, \mathrm{y} \text {, or } \mathrm{z} \text { direction }
$$

\section{Introduction}

A reusable rocket can be used several times and is expected to reduce its space transportation cost. ${ }^{1)}$ Vertical take-off and vertical landing ${ }^{2}$ (Fig. 1) is one of system concepts for reusable rockets. The representative reusable rocket is RVT (Reusable Vehicle Testing) ${ }^{3)}$ in JAXA (Fig. 2). Such vehicles should have slender configurations to achieve appropriate aerodynamics for its flight.

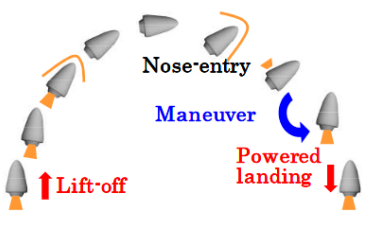

Fig. 1. Nose entry. $\left.{ }^{2}\right)$

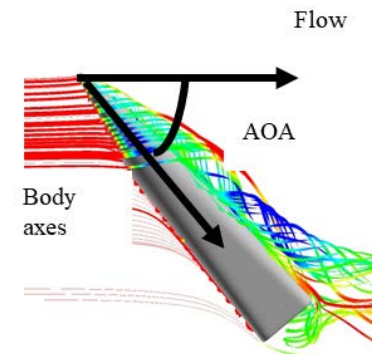

Fig. 3. Definition of angle of attack.

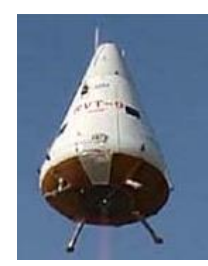

Fig. 2. RVT. ${ }^{3)}$

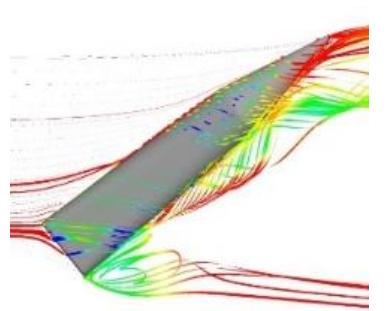

Fig. 4. Separated flow.

It is known that aerodynamic characteristics of such a slender body varies substantially at high angles-of-attack (AoAs) (Fig. 3 ), in which, flowfields become asymmetry because of large separations (Fig. 4), producing yaw force. ${ }^{4,5)}$ A large yaw force will destabilize the flight. In fact, an earlier study on a particular configuration $^{4)}$ reported large $\mathrm{C}_{\mathrm{Y}}$ (yaw force coefficient) and high flow asymmetry at high AoAs (50 degrees or more). 


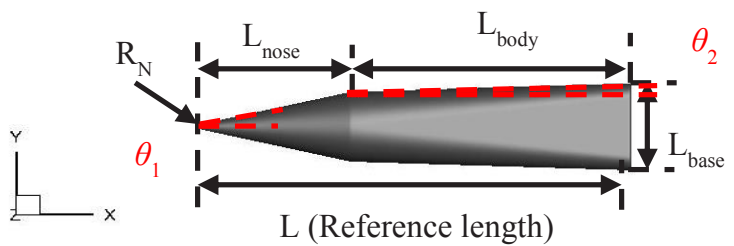

(a) Model A (baseline)

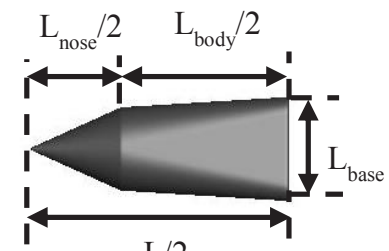

$\mathrm{L} / 2$

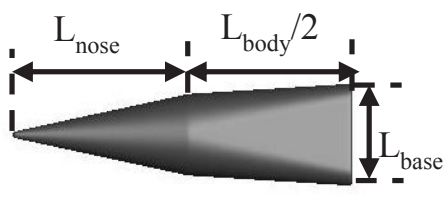

(c) Model C (half "body" length)

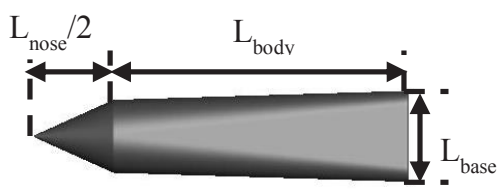

(d) Model D (half "nose" length)

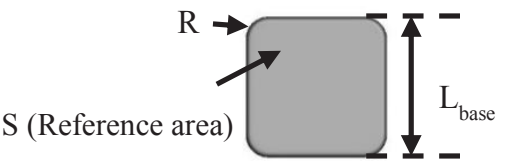

(e) Base view of Model A and B

Fig. 5. Configurations.

Table 1. Scale of the computational configurations.

\begin{tabular}{|c|c|c|}
\hline model & (a)Model A & (b)Model B \\
\hline $\mathrm{L}[\mathrm{mm}]$ & 307.02 & $\mathbf{1 5 3 . 5 1}$ \\
\hline $\mathrm{L}_{\text {nose }}[\mathrm{mm}]$ & 106.84 & $\mathbf{5 3 . 4 2}$ \\
\hline $\mathrm{L}_{\text {body }}[\mathrm{mm}]$ & 200.18 & $\mathbf{1 0 0 . 0 9}$ \\
\hline $\mathrm{L}_{\text {base }}[\mathrm{mm}]$ & 62.1 & 62.1 \\
\hline projected area $S\left[\mathrm{~mm}^{2}\right]$ & 3770.57 & 3770.57 \\
\hline $\mathrm{R}_{\mathrm{N}}[\mathrm{mm}]$ & 2.25 & $\mathbf{1 . 1 2 5}$ \\
\hline $\mathrm{R}[\mathrm{mm}]$ & 10 & 10 \\
\hline diameter of nose $\left[\mathrm{mm}^{2}\right]$ & 49.86 & 49.86 \\
\hline$\theta_{1}[$ degree $]$ & 12.21 & $\mathbf{2 4 . 3 8}$ \\
\hline$\theta_{2}[$ degree $]$ & 1.77 & $\mathbf{3 . 5 3}$ \\
\hline
\end{tabular}

As the first step of our study covering whole the flight envelop (and hence, all the possible angles of attack) of a reusable rocket, here we selected the 50 and 140 degrees (corresponding to $40 \mathrm{deg}$. if seen from the vehicle base) for particular reasons. Typically, the flowfields around flight vehicles are known to be either 1) symmetry and steady (at small angles), 2) asymmetry and steady (at moderate or high angles), or 3) asymmetry and unsteady (at very high angles,

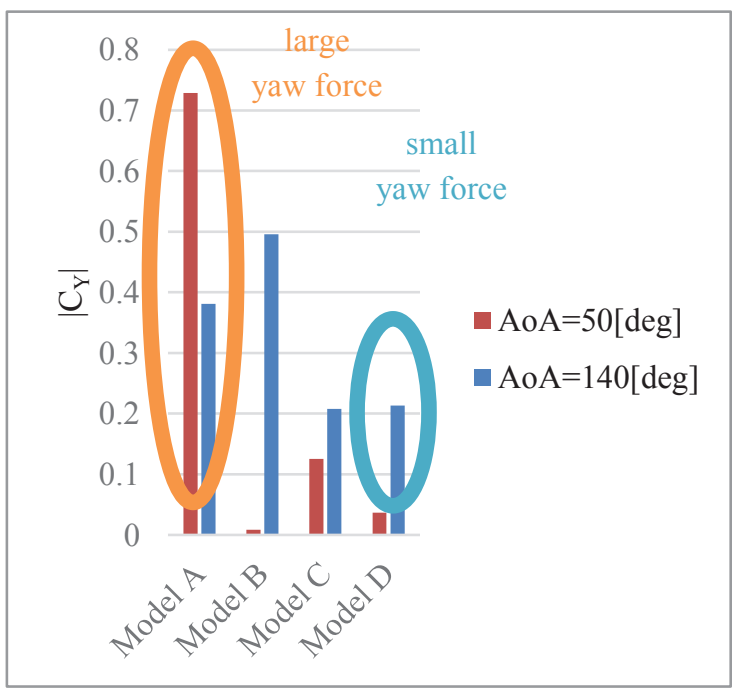

Fig. 6. Yaw force coefficients.

such as $90 \mathrm{deg}$ ). Among still not-yet-fully-understood flow physics in 2) and 3), we will pay a special attention to 2) because of cheaper computational cost required.

In the earlier study using RANS (Reynolds-AveragedNavier-Stokes), it was discovered that the flowfield around a slender configuration was clearly asymmetry, yet steady, at 50 and 140 degrees, whereas asymmetry and "unsteady" around 90 degrees, in which more sophisticated unsteady simulations 
such as LES (Large Eddy Simulation) would be necessary. Furthermore, the flowfields have not been fully explained or even studied at over 90 degrees (e.g., 140 degrees). These are the reasons why we focused on both the 50 and 140 AoAs in our present, RANS simulation study.

In addition, it is known that configurations such as fineness ratio and a half-apex-angle affect a yaw force. ${ }^{5)}$ Specifically, the blunt nose can reduce asymmetry. ${ }^{5)}$ Besides, the early studies $^{6,7)}$ show that the structure of the vortices around the body at high angle of attack. However, the detailed links between the flow asymmetry, $\mathrm{C}_{\mathrm{Y}}$, and the vehicle configurations particularly at more than 90 degrees of AoA have not been fully explained yet. We will numerically investigate the above-mentioned relations in this work.

\section{Analysis Configurations}

Figure 5 shows the all configurations which we investigated. In addition, the yaw force coefficients are shown in Fig. 6. The $\mathrm{C}_{\mathrm{Y}}$ of the Model $\mathrm{C}$ is larger in $140 \mathrm{AoAs}$ than $50 \mathrm{AoAs}$, like the Model B and the Model D. The Model D can reduce $\mathrm{C}_{Y}$ most, however, the reason was unclear why the $\mathrm{C}_{\mathrm{Y}}$ becomes small. We want to know the mechanism of the yaw force getting large. Therefore, we focused on the Model A and Model B especially, which indicated large yaw force coefficients here.

We use the configuration consisting of "nose cone" and "square body" parts ("Model A") as the baseline (Fig. 5 (a)), ${ }^{4,8)}$ and, Model B, which has different fineness ratio from that of Model A, proposed as in Fig. 5 (b). Model C has a half body length of Model A and Model D has a half nose length of Model A (Fig. 5 (c), (d)). In various configurations used in the early studies, ${ }^{9,10)}$ Model A is a promising candidate for reusable rockets because its separation point is rather fixed due to the square portion. In the earlier study, it is known that the squared body can reduce asymmetry of the flowfield than the cylinder body. ${ }^{8)}$ Moreover, it is also known that the face receiving the aerodynamic force is increased, and the lift force is generated by making the cross-sectional shape not a circle but a square. ${ }^{11)}$

In Fig. 5 (a), $R_{N}$ is the radius of nose tip, $L_{\text {nose }}$ is the nose-cone part length and $\mathrm{L}_{\text {body }}$ is the square-body part length. $\theta_{1}, \theta_{2}$ are the half apex angles of the nose and the body, respectively. In addition, $\mathrm{S}$ is the reference area as shown in Fig. 5 (e). It is determined as follow.

$$
S=\left(L_{\text {base }}-2 R\right)^{2}+\pi R^{2}+4 R\left(L_{\text {base }}-2 R\right)
$$

We investigated three different configurations based on Model A, however, in this paper, we focus only on two of these configurations which showed great yaw force. The size of Model A and Model B are shown in Table 1. Model B has half X-directional length of Model A. In Table 1., boldfaced types and gray backgrounds indicate that the parameters are different from Model A.

For instance, the half apex angle of the nose of Model B $\left(\theta_{1}=24.38[\mathrm{deg}]\right)$ is larger (almost twice) than that of Model A $\left(\theta_{1}=12.21[\mathrm{deg}]\right)$. Moreover, $\theta_{2}$ of Model A $\left(\theta_{2}=\right.$ $1.77[\mathrm{deg}])$ is smaller (almost half) than that of Model B $\left(\theta_{2}=\right.$ $3.53[\operatorname{deg}])$.

\section{Numerical Setup}

\subsection{Governing equation}

Governing equations are Navier-Stokes equations.

$$
\frac{\partial \boldsymbol{Q}}{\partial t}+\frac{\partial \boldsymbol{F}_{\boldsymbol{e}_{\boldsymbol{k}}}(\boldsymbol{Q})}{\partial x_{\mathrm{k}}}=\frac{1}{R_{e}} \frac{\partial \boldsymbol{F} v_{\boldsymbol{k}}}{\partial x_{k}}
$$

$\boldsymbol{Q}$ is a conservative variables, $\boldsymbol{F}_{\mathrm{e}}$ is inviscid variables, $\boldsymbol{F}_{\mathrm{v}}$ is viscous variables. These are as follows.

$$
\begin{gathered}
\boldsymbol{Q}=\left(\begin{array}{c}
\rho \\
\rho u_{l} \\
e
\end{array}\right), \quad \boldsymbol{F}_{\boldsymbol{e}}(\boldsymbol{Q})=\left(\begin{array}{c}
\rho \boldsymbol{u}_{\boldsymbol{k}} \\
\rho \boldsymbol{u}_{\boldsymbol{l}}+p \delta_{l k} \\
(e+p) \boldsymbol{u}_{\boldsymbol{k}}
\end{array}\right) \\
\boldsymbol{F}_{v}(\boldsymbol{Q})=\left(\begin{array}{c}
0 \\
\tau_{l k} \\
\boldsymbol{u}_{\boldsymbol{m}} \tau_{m k}+\frac{\kappa}{(\gamma-1) \operatorname{Pr}} \frac{\partial T}{\partial x_{k}}
\end{array}\right)
\end{gathered}
$$

Where subscripts $k, l$ and $m$ take 1, 2, 3 denoting the Cartesian coordinates. Then, $\rho, e, p, u_{\mathrm{i}}, \tau_{\mathrm{ij}}, \kappa, T, \gamma, \operatorname{Re}, \operatorname{Pr}$ and $\delta_{i j}$ are density, total energy per unit volume, pressure, velocity vector, stress tensor, coefficient of heat conduction, temperature, heat capacity ratio, Reynolds number, Prandtl number, and Kronecker delta, respectively. These have already been non-dimensionalized by

$$
\begin{gathered}
\rho=\frac{\widetilde{\rho}}{\rho_{\infty}}, e=\frac{\tilde{e}}{\rho_{\infty} a_{\infty}^{2}}, p=\frac{\tilde{p}}{\rho_{\infty} a_{\infty}^{2}}, u_{i}=\frac{\widetilde{u}_{i}}{a_{\infty}}, \\
\kappa=\frac{\kappa}{\kappa_{\infty}}, T=\frac{\tilde{T}}{T_{\infty}}, \operatorname{Re}=\frac{\rho_{\infty} a_{\infty} L}{\mu_{\infty}}=\frac{R_{e_{\infty}}}{M_{\infty}}, \operatorname{Pr}=\frac{C_{p} \mu_{\infty}}{\kappa_{\infty}}
\end{gathered}
$$

where tildes $(\sim)$ and infinity $(\infty)$ indicate dimensional quantities and freestream values, respectively. $L$ is the reference length, and $a_{\infty}$ is the freestream sound speed. Moreover, $\mu_{\infty}$ is the freestream viscosity coefficient and $C_{P}$ is the specific heat at constant pressure.

\subsection{Numerical methods}

In this study, we employ CFD solver "FaSTAR"12) developed at JAXA. All calculations are unsteady calculations. The time integration method is preconditioned LU-SGS along with dual-time-stepping and backward difference (second order), ${ }^{13)}$ and reconstruction method is GLSQ (second order). 14) The turbulence model is Spalart-Allmaras (SA) RANS model, ${ }^{15)}$ which is one of popular RANS turbulence models in aerospace engineering.

Note that more sophisticated turbulence models such as SST (Shear-Stress-Transport) $^{16)}$ or DDES (Delayed-DetachedEddy-Simulation) ${ }^{17)}$ would enhance the solution resolution, in expense of computational cost. The SA model, on the other hand, is in fact a good compromise between its accuracy and efficiency, particularly for the flow physics of our interest: The earlier study ${ }^{4,18)}$ on the similar configurations resulted in the 
similar solutions ( $\mathrm{C}_{\mathrm{Y}}$ and the flowfield) between $\mathrm{SA}$ and DDES models, as long as steady flowfields are obtained at low or moderate AoAs (typically 50 degrees or less).

Moreover, we employ Venkatakrishnan limiter as the slope limiter. We run calculations on supercomputer "JSS2" in JAXA.

\subsection{Computational grids}

We employ "HexaGrid"19) developed at JAXA. HexaGrid is a software which automatically generates grids based on hexahedron from STL data. Figure 7 shows the grid around the body. Outer boundary is set to $25 \mathrm{~L}$ away from the body. The origin is the nose tip, the body axis direction is the $\mathrm{X}$ axis and the direction which determines the yaw force is the $\mathrm{Y}$ axis. The $\mathrm{Z}$ axis is defined so that it is orthogonal to those two.

Furthermore, we use refinement boxes for arranging fine grids only in the region where vortices are formed (Fig. 8). Since the large vortices are known to the base at 50 degrees and near the nose at 140 degrees of AoA, according to our preliminary computations, we prepared large refinement boxes in these parts.

We decided the number of cells as in Table 2. We generated grids so that the first cell height almost satisfies $\mathrm{y}^{+}<1$.

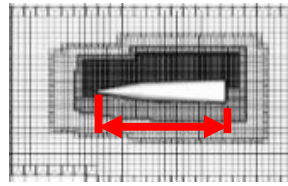

L

(a) Close-up view

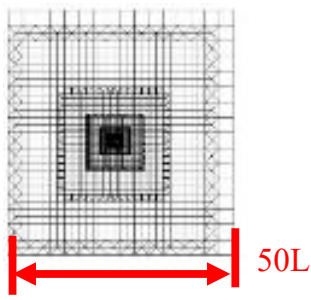

(b) Overview
Fig. 7. Computational grid.

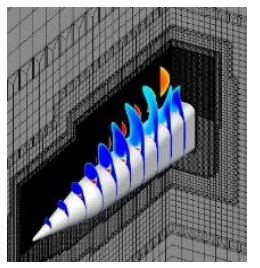

(a) $\mathrm{AoA}=50$ [degrees $]$

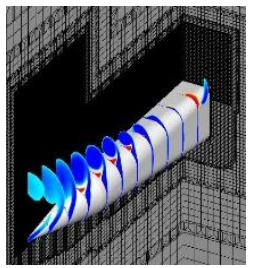

(b) $\mathrm{AoA}=140[$ degrees $]$
Fig. 8. Grid refinement and $\mathrm{X}$ Vorticity.

Table 2. Number of grids.

\begin{tabular}{|c|c|c|c|c|}
\hline model & \multicolumn{2}{|c|}{ (a)Model A } & \multicolumn{2}{c|}{ (b)Model B } \\
\hline AoA[deg] & 50 & 140 & 50 & 140 \\
\hline No. of cells & $1.75 \mathrm{M}$ & $8.95 \mathrm{M}$ & $4.01 \mathrm{M}$ & $5.44 \mathrm{M}$ \\
\hline $\mathrm{y}^{+}$ & 0.78 & 0.81 & 1.0 & 0.84 \\
\hline
\end{tabular}

\subsection{Numerical conditions}

The Mach number is 0.086 (flow speed is $30[\mathrm{~m} / \mathrm{s}]$ ), the freestream Reynolds number is set to $6.02 \times 10^{5}$. Moreover, the reference length is $0.307[\mathrm{~m}]$, the static pressure is set to $99920[\mathrm{~Pa}]$, and the static temperature is $291.15[\mathrm{~K}]$. These conditions are the same as that of wind tunnel test. ${ }^{8)}$ In this study, we defined the gravity center where $65 \%$ of total length from the nose tip.

\subsection{Definition of yaw force coefficient}

The yaw force coefficient is defined as follows.

$$
C_{\mathrm{Y}}=\frac{F_{y}}{\frac{1}{2} \rho_{\infty} U_{\infty}^{2} S}
$$

Where $U_{\infty}, S$, and $F_{\mathrm{y}}$ are the freestream velocity, the reference area (Base area), and the yaw force, respectively (Fig. 9).

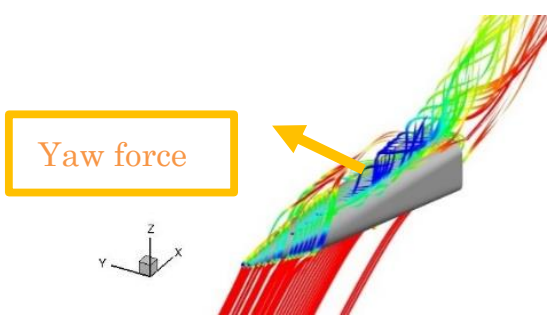

Fig. 9. Direction of Yaw force.

\section{Results and Discussion}

\subsection{Yaw force}

We obtain yaw force coefficients and visualized flowfields for two configurations (Model A and Model B) at 50 and 140 degrees of AoA.

From the yaw force coefficients in Table 3., the value is smallest in the cases of Model B at 50 degrees followed by Model A at 140 degrees of AoA.

\begin{tabular}{|c|c|c|c|c|}
\hline model & \multicolumn{2}{|c|}{ (a)Model A } & \multicolumn{2}{|c|}{ (b)Model B } \\
\hline AoA[deg] & 50 & 140 & 50 & 140 \\
\hline $\mathrm{C}_{\mathrm{Y}}$ & 0.729 & 0.381 & $8.36 \times 10^{-3}$ & 0.496 \\
\hline $\begin{array}{l}\text { Sym/ } \\
\text { Asym }\end{array}$ & Asym & Asym & Sym & Asym \\
\hline
\end{tabular}

Figures 10 and 11 indicate the visualization results of each case. The top figures show the on-surface pressure coefficient (viewed from $+\mathrm{z}$ ), and the bottom figures are the $\mathrm{X}$ vorticity. From these results, it can be said that the flowfield appears symmetric in the case of Model B at 50 degrees. On the contrary, apparent asymmetry can be observed in the cases of Model A at 50 degrees and Model B at 140 degrees of AoA. Therefore, the opposite trend appears between 50 degrees and 140 degrees of AoA. Now that we have observed a relation between the configurations and the yaw forces, in the next section, we will explain the difference of flowfields around the body between 50 degrees and 140 degrees of AoA.

\subsection{Relation between yaw force and flow field}

\subsection{1. $\mathrm{AoA}=50$ [degrees]}

\section{A. Model A (Baseline)}

In Fig. 12, the black lines indicate the streamlines around the body. From this figure, we can observe the evolution of the 
longitudinal vortices in the leeward side of the body. Furthermore, Fig. 13 shows $C_{P}$ and the streamlines on the body surface seen from the $+\mathrm{Z}$ direction.
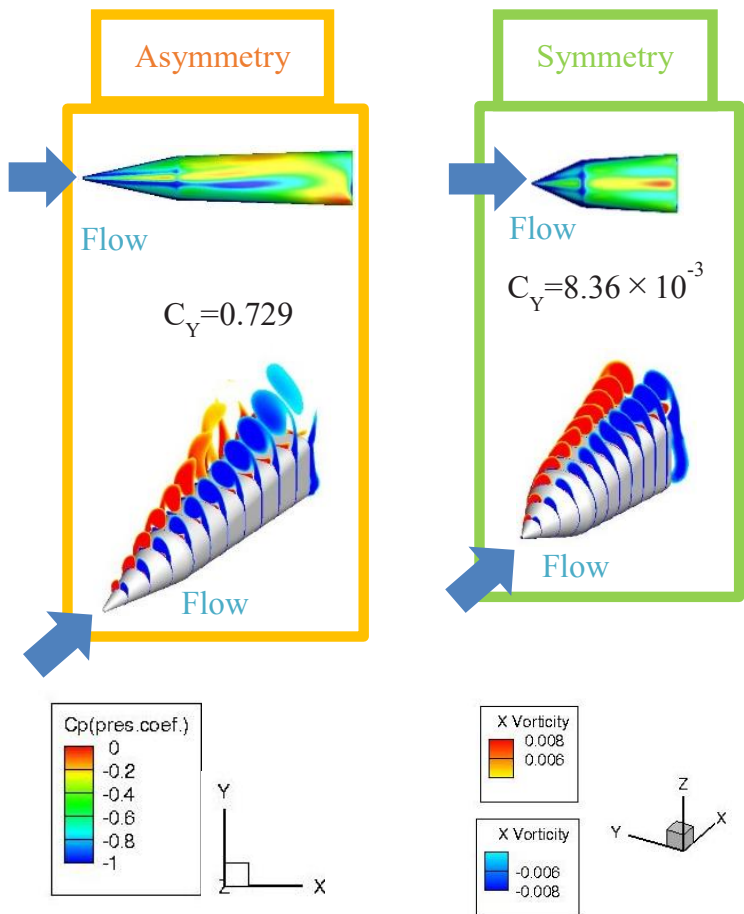

(a) Model A

(b) Model B

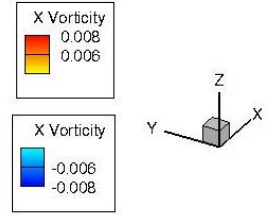

$($ AoA $=50[$ degrees $])$.

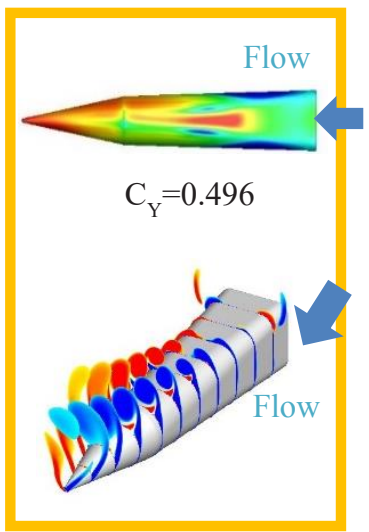

(a) Model A

(b) Model B

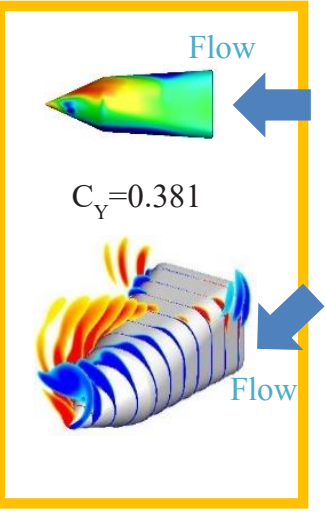

Fig. 11. Summery $(\mathrm{AoA}=140[$ degrees] $)$.

From Fig. 13, the flowfield is symmetry on the section of $10 \%$ of the total length from the nose tip, whereas asymmetry appears on the section of $70 \%$ and $90 \%$. In order to confirm to these, we show in Fig. 14 velocity vectors on the section of $10 \%, 70 \%$ and $90 \%$ of total length. These vectors are colored with X vorticity. From Fig. 14 (a), two symmetric vortices (V1, V2) are generated at the nose (10\%). However, the right vortex (V2) exists and the left vortex structure (V1) collapses near the gravity center $(70 \%)$ as evident in Fig. 14 (b). In addition, V2 on the 90\% section has grown from the $70 \%$ section in Fig. 14 (c). These results illustrate that the flowfield is symmetry on the $10 \%$ section but asymmetry on the $70 \%$ and $90 \%$ sections.

From these results, we can say that the vortex became

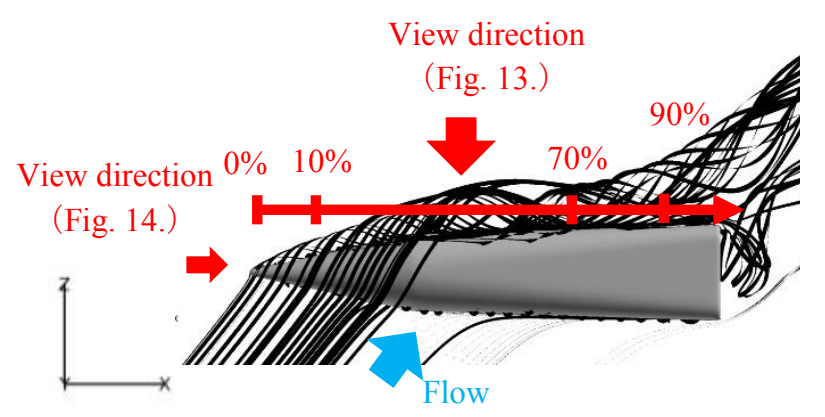

Fig. 12. Side view and streamlines.

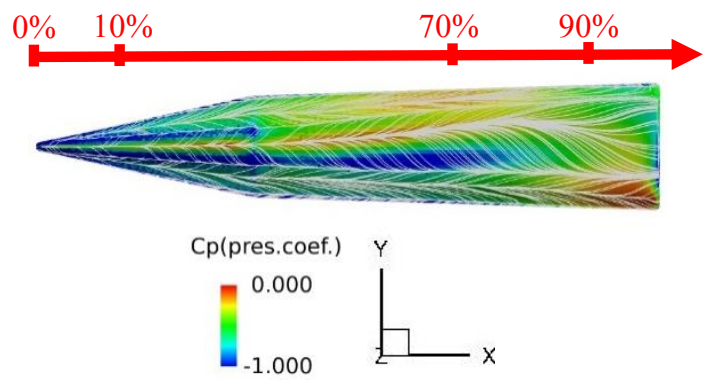

Fig. 13. $\mathrm{C}_{\mathrm{P}}$ and surface streamline (top view). $\mathrm{X}$ Vorticity

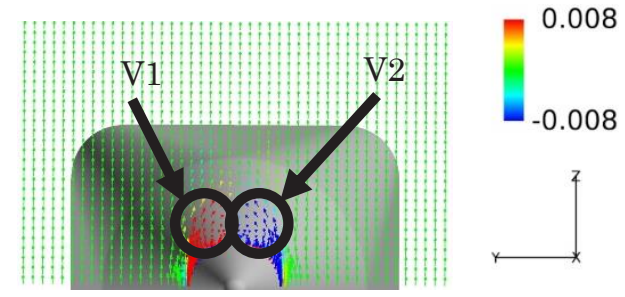

(a) $\mathrm{X}=10 \%$

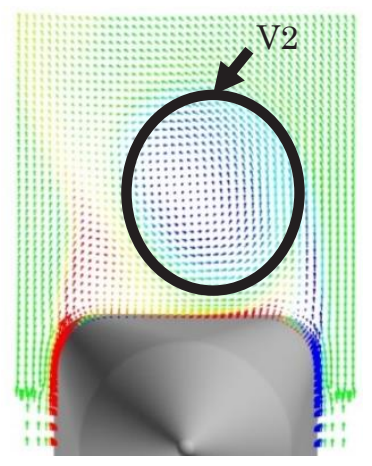

(b) $\mathrm{X}=70 \%$

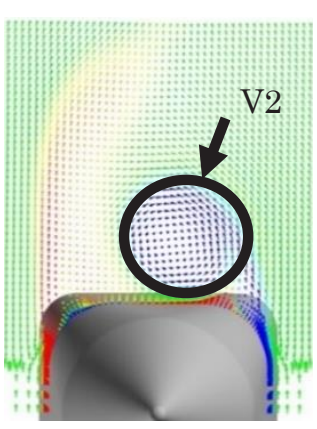

(c) $\mathrm{X}=90 \%$

Fig. 14. Velocity vectors with X Vorticity.

asymmetry in the downstream, and this caused the asymmetric $\mathrm{C}_{\mathrm{P}}$ distribution (Fig. 13). 
As a result, the large yaw force $\left(\mathrm{C}_{\mathrm{Y}}=0.729\right)$ was generated.

\section{B. Model B (Half Length)}

The flowfield appears totally symmetry in Fig. 15 which is confirmed from the on-surface $C_{P}$ distribution along with surface streamlines (in white) seen from the $+Z$ direction. Since the flowfields are symmetry on all, we show only the $70 \%$ section as the representative of these planes in Fig. 16, in which symmetry vortices (V1, V2) are observed. This is why yaw force $\left(\mathrm{C}_{\mathrm{Y}}=8.36 \times 10^{-3}\right)$ is negligibly small in this case.

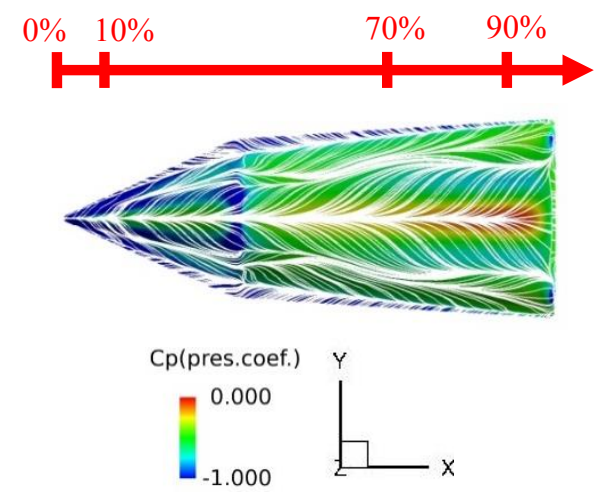

Fig. 15. $\mathrm{C}_{\mathrm{P}}$ and surface streamline (top view).

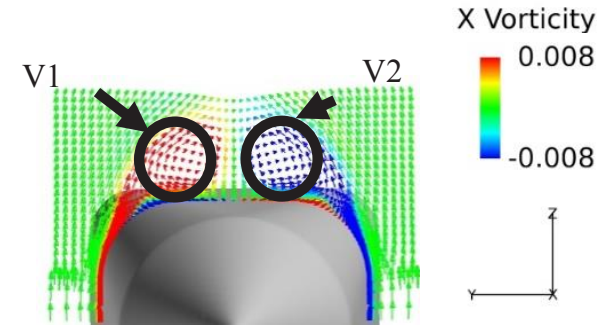

Fig. 16. Velocity vectors with $\mathrm{X}$ Vorticity $(\mathrm{X}=70 \%)$.

\section{Summary of $\mathbf{A o A}=\mathbf{5 0}$ [degrees]}

As we mentioned above, the flowfield of Model A becomes asymmetry clearly, although that of Model B is symmetry. This results in the larger $\mathrm{C}_{\mathrm{Y}}$ of Model A compared to Model B. As we mentioned in Section 2, Model B has a larger half apex angle from Model A, and the length of Model B is half of Model A. Thus, we can say that the configuration having a short nose and a large half apex angle would reduce yaw force. An earlier study $^{4)}$ on similar configurations also reported that the blunt nose can suppress asymmetry. In other words, our result is consistent with the literature for this AoA.

\subsection{2. $\mathrm{AoA}=140$ [degrees]}

\section{A. Model A (Baseline)}

To the best of the authors' knowledge there is no prior work which examined detailed aerodynamics at this AoA. Figure 17. indicates streamlines in which we can see vortices. Figure 18. shows the on-surface streamlines and $C_{P}$ distribution. $C_{P}$ in the downstream $70 \%$ and higher is totally symmetry as seen from this figure. We have focused on the three sections from the upstream to the downstream in the previous sections. In 140 degrees of AoA, however, we omit section of $10 \%$ because vortex does not exist. As shown in Fig. 19 (a), two asymmetric vortices (V3, V4) can be seen on the $70 \%$ section. These vortices are constituted of the flows which passed through the base (Fig. 17), and thus, these are different from the two vortices $(\mathrm{V} 1, \mathrm{~V} 2)$ which are generated at nose tip in 50 degrees of AoA. On the $90 \%$ section, the vortex structure (V3) collapses while the vortex (V4) is further developed, compared to the $70 \%$ section. Therefore, inspite of the different generation mechanisms of vortices, asymmetry can be seen in the downstream at this $140 \mathrm{deg}$. AoA, as in the case of 50 degrees. For this reason, the yaw force $\left(C_{Y}=0.381\right)$ is generated, which is smaller than $\mathrm{C}_{\mathrm{Y}}=0.729$ at 50 deg., but still significant.

\section{B. Model B (Half Length)}

Visualized results are displayed in Figs. $20-22$ as in the other cases. Asymmetry can be seen clearly in Fig. 21. Furthermore, the small asymmetry vortices (V3, V4) are produced near the body as evident in Fig. 22 (a). In Fig. 22 (b), only V4 exists whereas V3 already decayed. As a result, the $\mathrm{C}_{\mathrm{P}}$ distribution becomes asymmetry from the vicinity of the gravity center $(65 \%)$ to the nose $(70 \%$ and $90 \%)$ (Fig. 21$)$. These results can be also confirmed from the development of the large yaw force $\left(\mathrm{C}_{\mathrm{Y}}=0.496\right)$.

Now, we discuss the reason why the flowfield is asymmetry clearly at the nose. The swirling flows appear in three places, the upstream and the downstream (Fig. 19, Fig. 22, V3, 4), and the flow especially gathers in the forward (downstream) of the body. From this fact and the visualization result, we can say that the nose blunt body which is rear-positioned when $\alpha>$ $90^{\circ}$ raises asymmetry of the flowfield. Let us briefly explain the reason of the asymmetric vortices by using Fig. 23. Figure 23 shows the relation between nose bluntness and asymmetry. In this figure, nose-sharp configuration (Model A) (i.e., sharp "trailing edge" when $\alpha>90^{\circ}$ ) and a nose-blunt configuration (Model B) (i.e., blunt "trailing edge" when $\alpha>90^{\circ}$ ) are illustrated. It is known that fluids tend to flow along a sharp trailing edge, whereas they can separate more easily and become asymmetry in the case of a blunt trailing edge, such as Karman vortices. In summary, we consider the reason of asymmetric flowfields of the Model B is that the "nose cone" part (i.e., downstream part if $\mathrm{AoA}>90^{\circ}$ ) behaves as a blunt "trailing edge" in this study.

\section{Summary of AoA $=\mathbf{1 4 0}$ [degrees]}

$\mathrm{C}_{\mathrm{Y}}$ of Model $\mathrm{B}\left(\mathrm{C}_{\mathrm{Y}}=0.496\right)$ is larger than that of Model A $\left(C_{Y}=0.381\right)$. Thus, we have obtained the opposite results from those of 50 degrees. That is, Model B generates the more asymmetric flowfield at 140 degrees than that at 50 degrees, whereas the flowfield is symmetry at 50 degrees. Moreover, the flowfields are asymmetry around both configurations at 140 degrees. In other words, asymmetry can occur easily over 90 degrees. The reason is that the blunt trailing edge makes vortex asymmetry in downstream.

Moreover, $\mathrm{C}_{\mathrm{Y}}$ of the Model $\mathrm{C}$ in 140 AoAs is as large as the Model D. From this result, the bluntness of body part also effects asymmetry and yaw force. The Model B and the Model $\mathrm{C}$ have blunt bodies and this is one of the reason of asymmetry. 


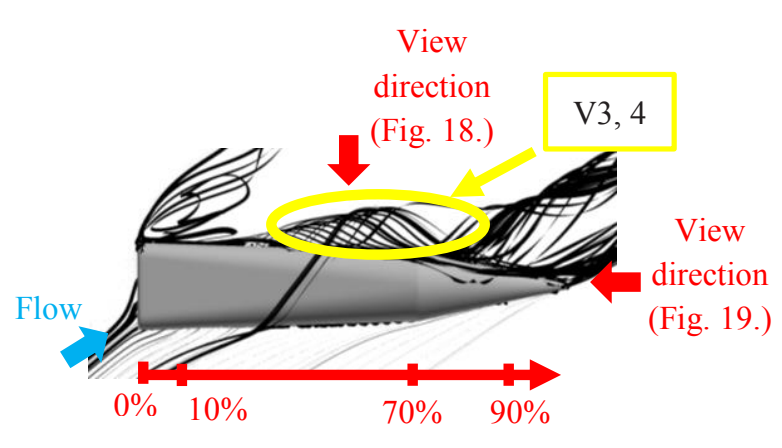

Fig. 17. Side view and streamlines (Model A).

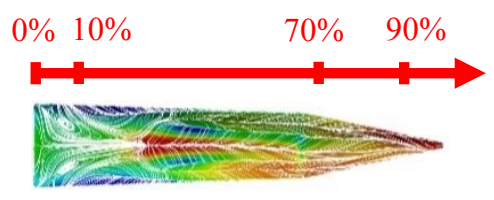

Fig. 18. $\mathrm{C}_{\mathrm{P}}$ and surface streamline (top view of Model $\mathrm{A}$ ).

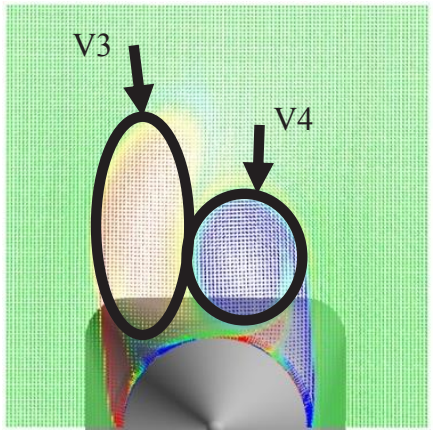

(a) $\mathrm{X}=70 \%$

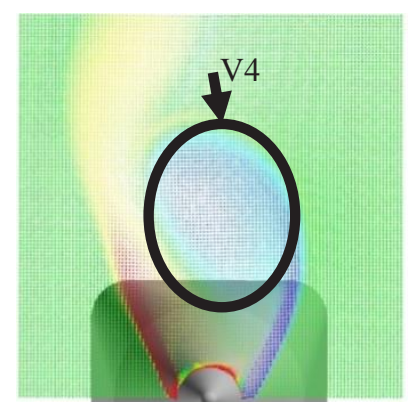

(b) $\mathrm{X}=90 \%$

Fig. 19. Velocity vectors with X Vorticity (Model A).

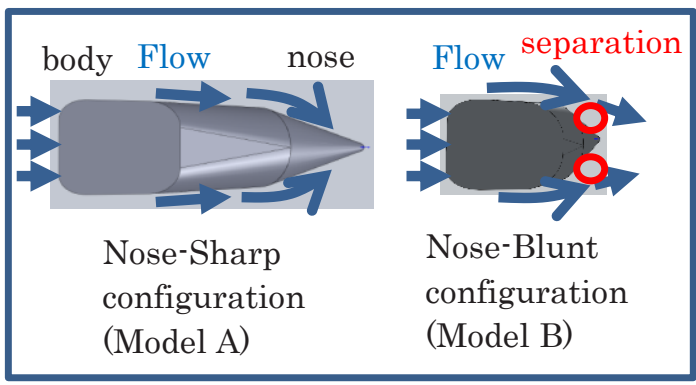

Fig. 23. Nose-Bluntness and asymmetry (when $\alpha>90^{\circ}$ ).

\section{Conclusions}

In this study, we numerically investigated the details of

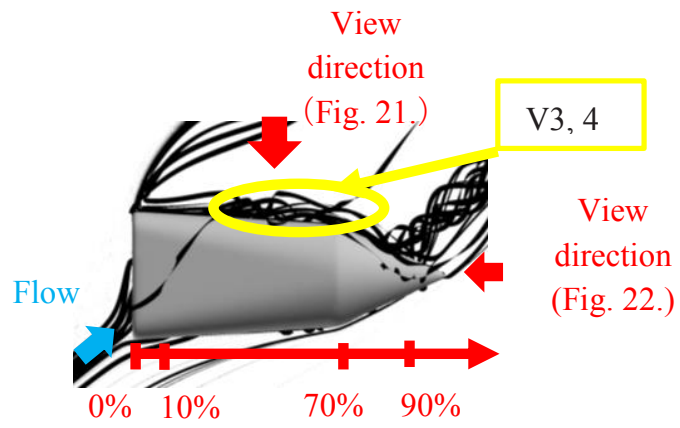

Fig. 20. Side view and streamlines (Model B).

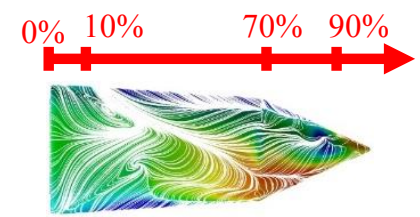

Fig. 21. $\mathrm{C}_{\mathrm{P}}$ and surface streamline (top view of Model $\mathrm{B}$ ).

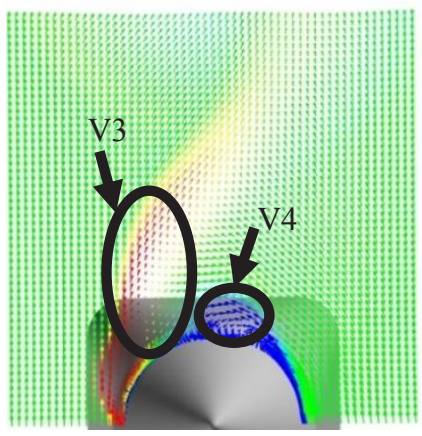

(a) $\mathrm{X}=70 \%$

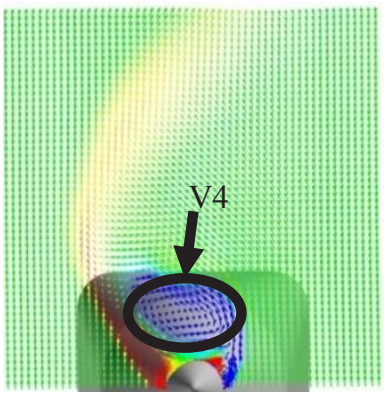

(b) $X=90 \%$

Fig. 22. Velocity vectors with X Vorticity (Model B).

flowfields around potential reusable rocket configurations at high angles-of-attack. Then, we obtained the results as follows by comparing different configurations.

- At 50 degrees, the longer the body becomes, the more asymmetry of two vortices is developed. From this result, we confirm that the blunt configuration having the short total length reduces asymmetry.

- At 140 degrees, the configuration which has the short total length increases asymmetry; this is the opposite trend to that at 50 degrees. This is because flowfields increase asymmetry while passing along the blunt nose (i.e., rear part when $\alpha>90^{\circ}$ ). Moreover, the blunt body is also one of the reason of asymmetry. 
Considering these results, we explain why the Model D best suppressed the asymmetry. The Model D has a half-length of the nose part and the same length of the body part as the model A (baseline). The half long nose reduced asymmetry at 50 AoA, whereas the long body kept symmetry at 140 AoA (Table 4.).

Table 4. Results of the Model D.

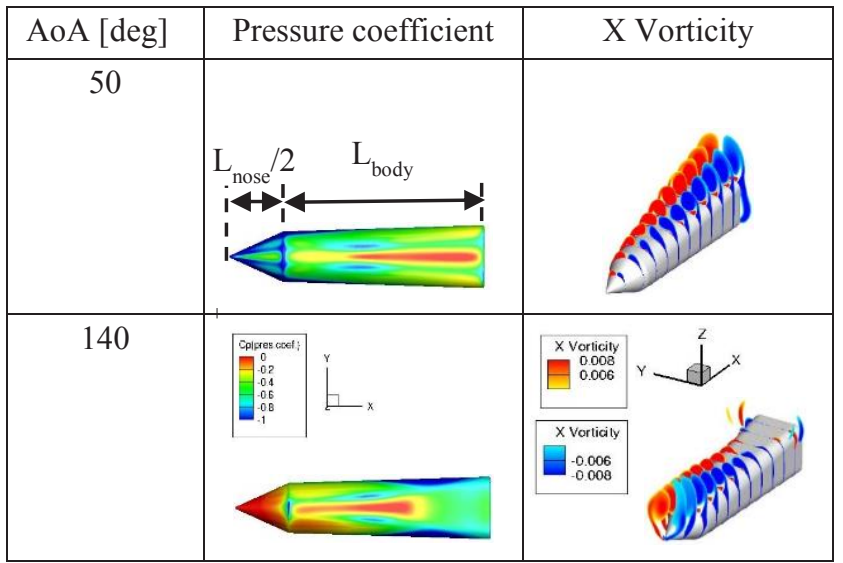

We conclude from these results as follows: It is newly discovered that blunt configurations which have small fineness ratios likely to show asymmetry over 90 degrees.

We have selected the present Reynolds number from the wind test in the similar work (in which the flow simulation and the experiment matches well each other). ${ }^{8)}$ It is already reported, however, that the Reynolds number can significantly affect $\mathrm{C}_{\mathrm{Y}}$ and the asymmetry of the flowfield, particularly at turbulent transition (as in this work). ${ }^{20)}$ Thus, we would like to simulate the flowfields at different Reynolds numbers (e.g., flight Reynolds number) in the future. Such a work on Reynolds effects is deferred to the future work.

\section{Acknowledgments}

These calculations were performed on JAXA Supercomputer System generation 2 (JSS2). We used FaSTAR as the fluid solver. The authors appreciate the feedback offered by Dr. Taro Shimizu of Japan Aerospace Exploration Agency and Mr. Junya Aono of Research Center of Computational Mechanics, Inc. Advices and comments given by colleagues of our laboratory have been a great help in this paper.

\section{References}

1) Kawado, H., Ishikawa, K., Niu, K., Takida, J., Obase, K, and Fukuda, Y.: Status of Research and Development Activities on Reusable Launch Vehicle, Technical Report of Mitsubishi Heavy
Industries, $\quad \mathbf{5 1}, \quad$ No.4, 2014 https://www.mhi.co.jp/technology/review/pdf/514/514044.pdf (accessed February 8, 2017) (in Japanese).

2) Kei, K., Takagi, R., Oyama, S., Fujii, T., and Yamamoto, S.: Computational Analysis of Aerodynamic Characteristics during the Launch and Return of the Reusable Observation Vehicle, 23rd Symposium on Computational Fluid Dynamics, 2009 (in Japanese).

3) About the third reusable rocket experiment machine taking off and landing experiment (RVT-9) in JAXA,

http://www.jaxa.jp/press/2003/11/20031112 rvt j.html (January 7, 2017) (in Japanese).

4) Aogaki, T., Kitamura, K., and Nonaka, S.: Computational Study of Aerodynamic Characteristics of Reusable Rocket at High-Angle-ofAttack, AIAA Paper 2017-1212, 2017.

5) Ericsson, L. E. and Reding, J. P.: Steady and Unsteady VortexInduced Asymmetric Loads on Slender Vehicles, AIAA J., 18 (1981),pp.97-109.

6) Jia, M., Watanuki, T., and Kubota, H.: The Visualization of Separated Flow Over Slender Bodies at High Angle of Attack, Transactions of the Visualization Society of Japan, 14 (1994), pp.3746, (in Japanese).

7) Cummings, R., Forsythe, J., Morton, S., and Squires, K.: Computational Challenges in High Angle of Attack Flow Prediction, Progress in Aerospace Science, 39, (2003), pp.369-384.

8) Kinami, T.: Aerodynamic Characteristics of Slender Bodies at Turnover, graduation thesis, Yokohama National University, Kanagawa, Japan, 2016, (in Japanese).

9) Fujimoto, K.: Computational Aerodynamic Analysis of Capsule Configurations Toward the Development of Reusable Rockets, Journal of Spacecraft and Rockets, 43 (2006), pp.77-83.

10) Imamura, Y.: Attitude Maneuver of VTOL Rocket in Landing Phase, master's thesis, Tokyo University, Tokyo, Japan, 2008.

11) Nakamura, M., Nonaka, S., and Inatani, Y.: Aerodynamics of Reusable VTOL Rocket in Return Flight, Proceedings of 58th Space Sciences and Technology Conference, 2014 (in Japanese).

12) Hashimoto, A., Murakami, K., Aoyama, T., Ishiko, K., Hishida, M., Sakashita, M., and Lahur, P.: Toward the Fastest Unstructured CFD Code "FaSTAR", AIAA Paper 2012-1075, 2012.

13) Kitamura, K., Shima, E., Fujimoto, K., and Wang, Z. J.: Performance of Low-Dissipation Euler Fluxes and Preconditioned LU-SGS at Low Speeds, Commun. Comput. Phys., 10 (2011), pp. 90-119.

14) Shima, E., Kitamura, K., and Haga, T.: Green-Gauss/WeightedLeast-Squares Hybrid Gradient Reconstruction for Arbitrary Polyhedra Unstructured Grids, AIAA J., 51 (2013), pp.2740-2747.

15) Spalart, P. and Allmaras, S.: A One-Equation Turbulence Model for Aerodynamics Flows, AIAA Paper 1992-439, 1992.

16) Menter, F. R.: Two-Equation Eddy-Viscosity Turbulence Models for Engineering Applications, AIAA J., 32 (1994), pp. 1598-1605.

17) Spalart, P. R., Deck, S., Shur, M. L., Squires, K. D., Strelets, M. K., and Travin, A.: A New Version of Detached-Eddy Simulation, Resistant to Ambiguous Grid Densities, Theoretical and Computational Fluid Dynamics, 20 (2006), pp. 181-195.

18) Aogaki, T., Kitamura, K., and Nonaka, S.: Numerical Analysis of Aerodynamic Characteristics of Reusable Rocket at High-Angle-ofAttack, 48th ANSS, Kanazawa, Japan, 2016 (in Japanese).

19) Hashimoto, A., Murakami, K.., Aoyama, T., Yamamoto, K., and Murayama, M.: Drag Prediction on NASA CRM Using Automatic Hexahedra Grid Generation Method, Journal of Aircraft, 51 (2014), pp.1244-1267.

20) Kubota, H., Arai, I., and Matsuzawa, M.: Flat Spin of Slender Bodies at High Angles of Attack, Journal of Spacecraft, 20 (1983), pp.108114. 